\title{
Atenuação de múltiplas utilizando as transformadas f-k e Radon
}

\author{
Wilker E. Souza, Rafael R. Manenti \& Milton J. Porsani
}

Copyright 2016, SBGf - Sociedade Brasileira de Geofísica.

Este texto foi preparado para a apresentao no VII Simpsio Brasileiro de Geofsica, Ouro Preto, 25 a 27 de outubro de 2016. Seu contedo foi revisado pelo Comit Tcnico do VII SimBGf, mas no necessariamente representa a opinio da SBGf ou de seus associados. proibida a reproduo total ou parcial deste material para propsitos comerciais sem prvia autorizao da SBGf.

\section{Abstract}

Multiples attenuation is always a subject of interest in seismic reflection field. This kind of reflection tends to overlay deeper primary waves present on data. With that in mind, this paper brings the use of $f-k$ transform and Radon transform for multiple reflections attenuation. Both methods work with events inclination, each one with its own particularity. Results show that both methods are good for this kind of application and a comparison between them is made.

\section{Introdução}

O processamento de dados sísmicos visa tratar os dados adquiridos em campo (terra ou mar) de modo que se obtenha uma imagem (seção empilhada) de boa qualidade e que melhor represente a realidade geológica em subsuperfície. Os dados sísmicos são contaminados durante sua aquisição por ruídos aleatórios e coerentes, como as reflexões múltiplas da energia sísmica no fundo do mar numa aquisição sísmica marinha.

Este artigo tem por objetivo a determinação de um fluxo ótimo para atenuação de múltiplas de superfície aplicado a um dado real 2D com o auxílio de transformadas. Será analisado a aplicação de duas metodologias de atenuação de múltiplas de superfície. $\mathrm{Na}$ classe de metodologias que se baseiam na diferença de comportamento entre múltiplas e primárias, serão analisadas a filtragem $f \quad k$ (Zhou; Greenhalgh, 1994), (Houston, 1998) e (Lokshtanov; Helle, 1992) e a filtragem Radon parabólica (Oliveira et al., 2007) e (Abbad et al.,2011).

\section{Filtro f-k}

A transformada FK consiste na aplicação da transformada de Fourier 2D, passando o dado, inicialmente no domínio tx (tempo-distância) para o domínio f-k (frequência-número de onda) (Yilmaz, 1987). Esta transformada possui uma característica bastante útil que é a segregação de eventos com diferentes inclinações dentro do sismograma. Para a atenuação de múltiplas utilizando filtro $\mathrm{f}-\mathrm{k}$, é necessário que se faça um campo de velocidades "errôneo". A ideia geral é que o usuário, ao fazer a análise de velocidades, escolha pontos intermediários entre a múltipla, que possui velocidade em torno de $1500 \mathrm{~m} / \mathrm{s}$, e as ondas primárias do resto do CMP. A consequência deste procedimento pode ser vista na Figura 1, onde as ondas primárias, por possuírem velocidades mais baixas que as escolhidas, serão sobrecorrigidas, ficando com sua curvatura para cima. Já os demais refletores, por possuírem velocidades maiores que as escolhidas, serão subcorrigidos, ficando com suas inclinações para baixo. Após este procedimento, é aplicada então a transformada $\mathrm{f}-\mathrm{k}$, onde as reflexões múltiplas estarão segregadas para a direita do painel (número de onda positivo) e as demais reflexões estarão segregadas na parte esquerda do painel (número de onda negativo), como podemos observar na Figura 3. Desta maneira, selecionamos um polígono e o atenuamos no painel f-k. Podemos observar na Figura 4 uma família CMP após este procedimento, e nela observamos que as múltiplas foram devidamente atenuadas. Após a aplicação do filtro, faz-se a correção NMO inversa, recuperando o CMP para o seu formato original, onde posteriormente será feita outra análise de velocidades.

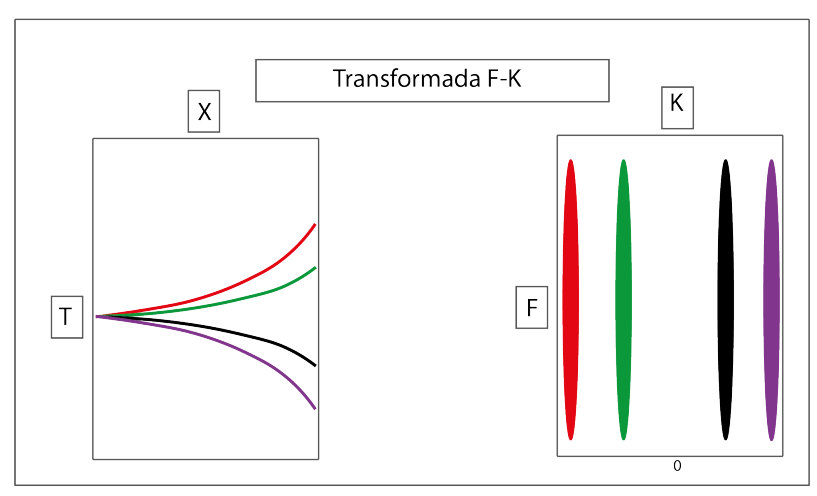

Figure 1: Esquema mostrando funcionamento da transformada $\mathrm{f}-\mathrm{k}$.

\section{Transformada Radon}

A transformada de Radon é constituída em dois passos: a soma do dado sísmico ao longo de uma curva e o mapeado dessa soma em um novo domínio chamado Radon. De maneira similar a filtragem fk, em que o dado é mapeado para o domínio fk, na filtragem Radon, o dado é convertido através da transformada de Radon, onde os eventos indesejáveis são removidos através de filtragem. A transformada consiste em uma soma em um percurso parabólico aplicada no dado após correção NMO, definida matematicamente por Verschuur (2006):

$$
m(q, \tau)=\int_{-\infty}^{+\infty} d\left(x, t=\tau+q x^{2}\right) d x
$$

onde $m(q, \tau)$ é a transformada Radon Parabólica direta, $x$ e t são as variáveis de entrada e o parâmetro q é, frequentemente, referido como o parâmetro de curvatura. Isto significa que os eventos no domínio tempo-distância que tem uma forma parabólica serão mapeados em pontos no domínio Radon parabólico, ver Figura 2. 
Este método deu um enorme impulso para aplicação na atenuação de múltiplas pela discriminação do sobretempo (Verschuur, 2006). Para a aplicação da filtragem Radon, o dado, no domínio CMP, foi corrigido de NMO e aplicado a transformada. Verifica-se que as reflexões múltiplas no domínio Radon diferenciam das reflexões. Um polígono é desenhado na região que contemplam as múltiplas e posteriormente o filtro é aplicado utilizando este polígono. A Figura 5 ilustra a construção do polígono e a Figura 6, o resultado da atenuação.

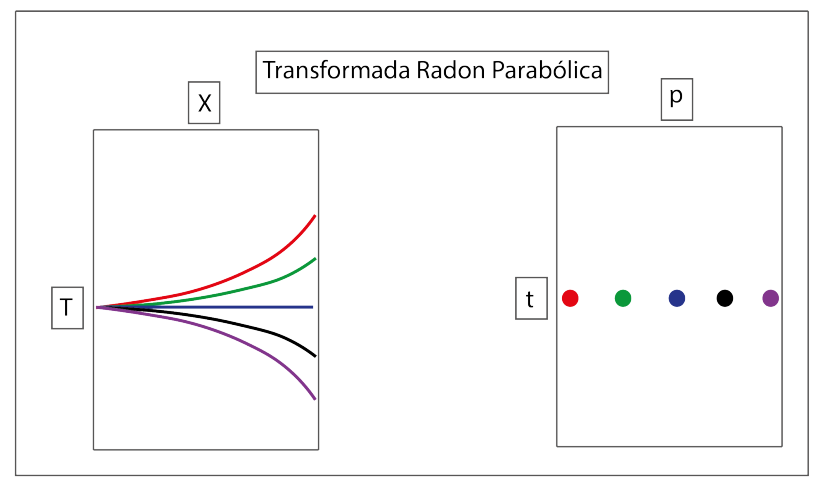

Figure 2: Esquema mostrando funcionamento da transformada Radon.

\section{Metodologia e Resultados}

Para testes, foi utilizado um trecho da linha 214-RL-0266 da bacia do Jequitinhonha, localizada ao sul da Bahia. A linha possui 120 canais, espaçados de $25 \mathrm{~m}$ entre eles e tendo como lanço 0-150-3150 m. A distância entre tiros foi de $25 \mathrm{~m}$ e o dado possui 1751 amostras com intervalos de amostragem de $4 \mathrm{~ms}$, totalizando um tempo de registro de 7 s. A cobertura CMP é de $6000 \%$.

Neste dado, com exceção dos métodos de filtragem, todos os outros passos do fluxograma foram seguidos da mesma forma, para fazer uma comparação mais justa entre os métodos. Foi aplicado um mute para eliminar a onda direta, seguida da aplicação de OAR (offset amplitude recovery) utilizando um campo de velocidades previamente obtido. Seguindo estes métodos foi aplicada uma deconvolução de consistência superficial, com o objetivo de comprimir o pulso do dado e aumentar a resolução do mesmo. Após isso foram aplicados os métodos de atenuação de múltiplas (neste momento foi separado o trabalho em duas vertentes), seguido da análise de velocidades, empilhamento e migração.

Como podemos observar a seção empilhada na Figura 8, o dado é fortemente contaminado com reflexões múltiplas da primária. Para este trabalho utilizamos dois métodos de atenuação de múltiplas: filtro f-k; e transformada Radon. $\mathrm{Na}$ Figura 7 observamos o CMP 720 original em (a) e filtrado com os métodos f-k e Radon, em (b) e (c), respectivamente. Ao analisarmos as figuras, vemos que as reflexões múltiplas, localizadas no tempo entre 2300 e $2400 \mathrm{~ms}$, foram atenuadas satisfatoriamente por ambos os métodos. Para comparação final, podemos observar a seção empilhada pós f-k em Figura 9 e a seção empilhada pós Radon em Figura 10. Vemos também que ambas possuem resultados satisfatórios, não tendo um método se destacando mais em relação ao outro.

\section{Conclusões}

Sabendo que o objetivo do processamento sísmico e gerar uma imagem de qualidade, se aproximando o máximo possível da seção geológica em subsuperfície. $O$ processamento da linha sísmica 214-RL-0266 apresentou resultados satisfatórios gerando uma seção sísmica de qualidade com nítidos refletores e atenuada de múltiplas de longo período. Podemos observar que os resultados alcançados utilizando as filtragens F-K e Radon para atenuação de múltiplas foram consideradas suficientes. Os resultados das duas filtragens foram semelhantes e tiveram uma melhor atenuação em regiões de lâmina d'água profunda.

\section{Agradecimentos}

Os autores agradecem à BP/PABIP, INCT-GP/CNPq/MCT e PETROBRAS pelo apoio financeiro e à LANDMARK pelas licenças disponibilizadas para o grupo CPGG/UFBA.

\section{Referências}

Abbad, B.; Ursin, B.; Porsani, M. J., 2011. A fast, modified parabolic radon transform. Society of Exploration Geophysicists, v. 76, n. 1, p. V11-V24, Jan.

Houston, L. M., 1998. Multiple suppression using a local coherence filter. Society of Exploration Geophysicists, v. 63 , n. 2, p. 652-659, Mar.

Lokshtanov, D.; Helle, H., 1992. Efficient f-k modeling approach in multiple elimination. Society of Exploration Geophysicists - Expanded Abstracts, v. 11, p. 1077-1080.

Oliveira, A. S.; Santos, J. R. S. B.; LIMA, G. R., 2007. Truevelocity Radon Filter. Society of Exploration Geophysicists - Expanded Abstracts, v. 26, p. 2530-2534.

Verschuur, D.J., A. J. Berkhout, C.P.A. Wapenaar, 1992, Adaptive surface-related multiple elimination. Geophysics, 57, 1166-1177.

Verschuur, D. J., 2006. Seismic multiple removal techniques. The Netherlands: European Association of Geocientists and Engineers, 191 p.

Yilmaz, Ö., 1987, Seismic data processing: SEG, Tulsa.

Zhou, B.; Greenhalgh, S. A., 1994 Wave-equation extrapolation-based multiple attenuation: 2-D filtering in the $\mathrm{f}-\mathrm{k}$ domain. Society of Exploration Geophysicists, v. 59, n. 9, p. 1377-1391, Sep. 


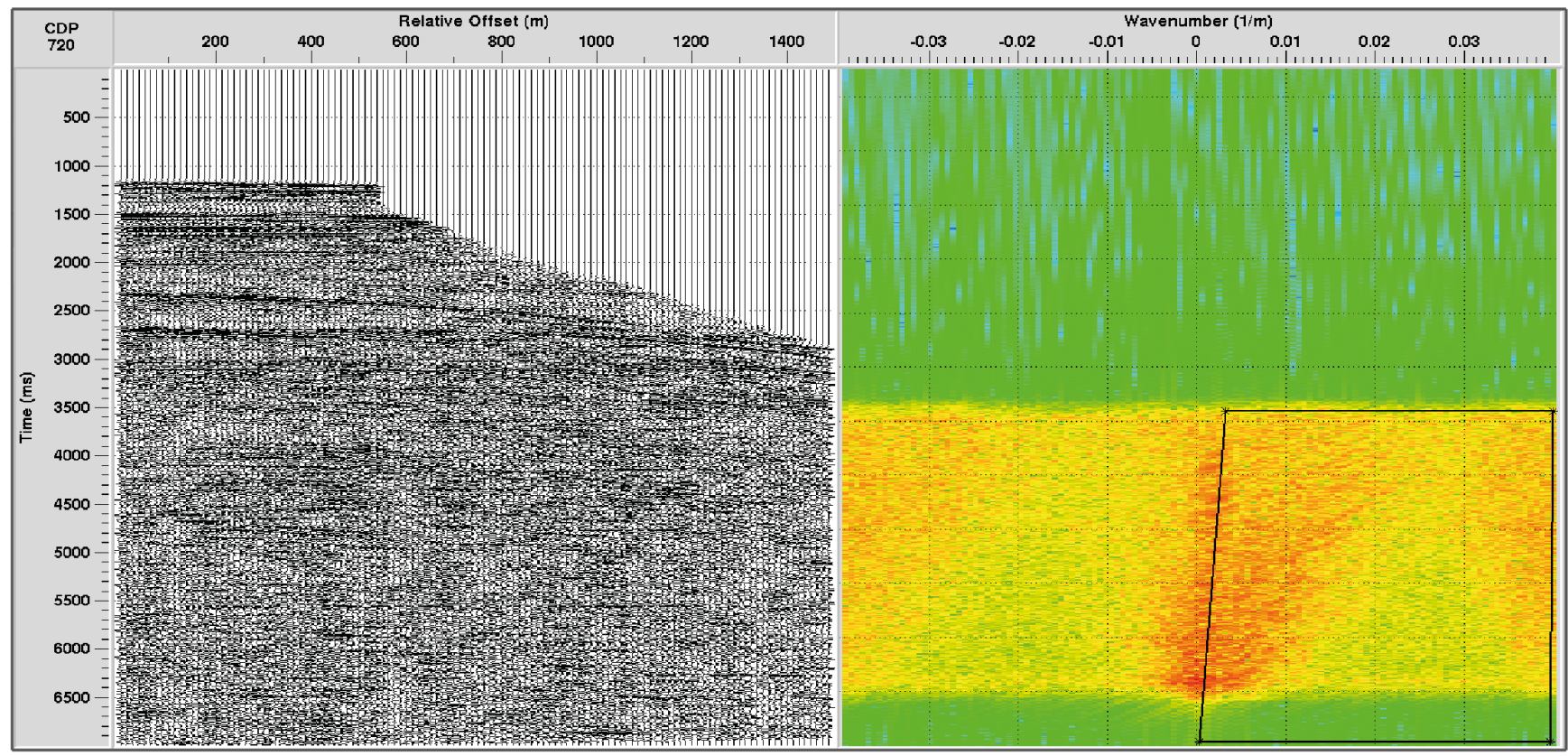

Figure 3: CMP 720 com corrigido com campo de velocidades intermediária e seu respectivo espectro f-k com polígono de atenuação exposto.

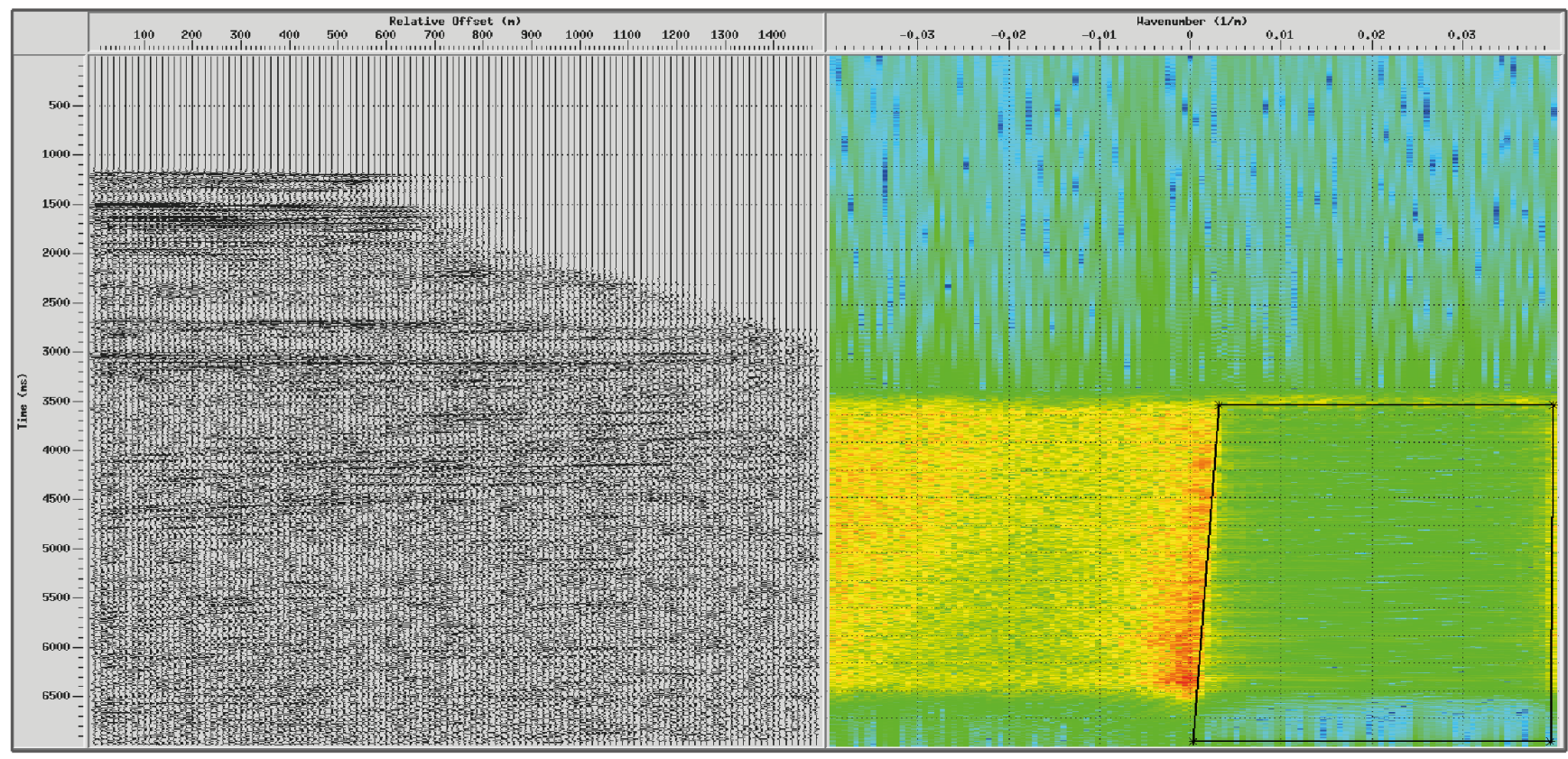

Figure 4: CMP 720 com atenuado e corrigido com campo de velocidades intermediária e seu respectivo espectro f-k com polígono de atenuação exposto. 


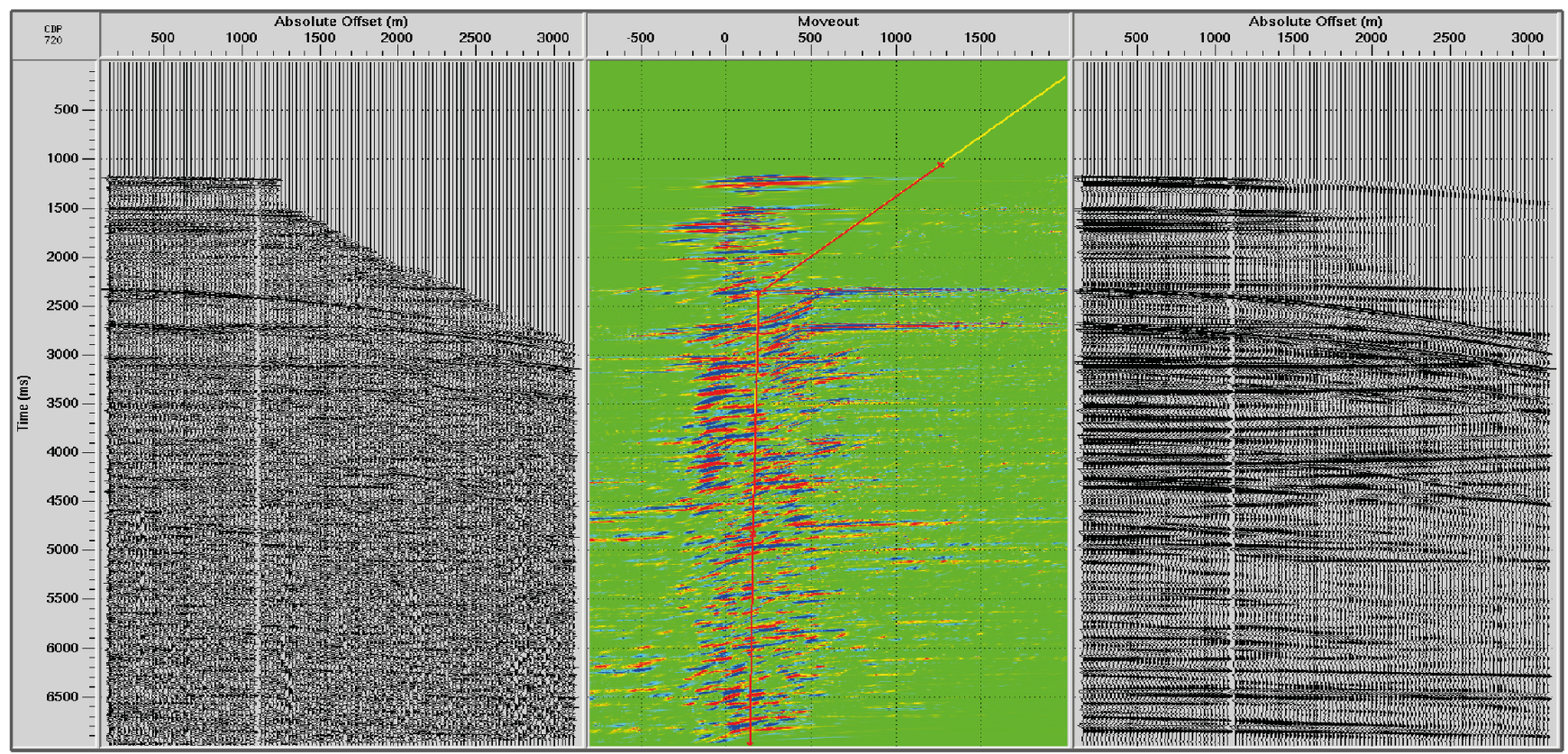

Figure 5: CMP 720 com corrigido cde NMO e seu respectivo espectro Radon com polígono de atenuação exposto.

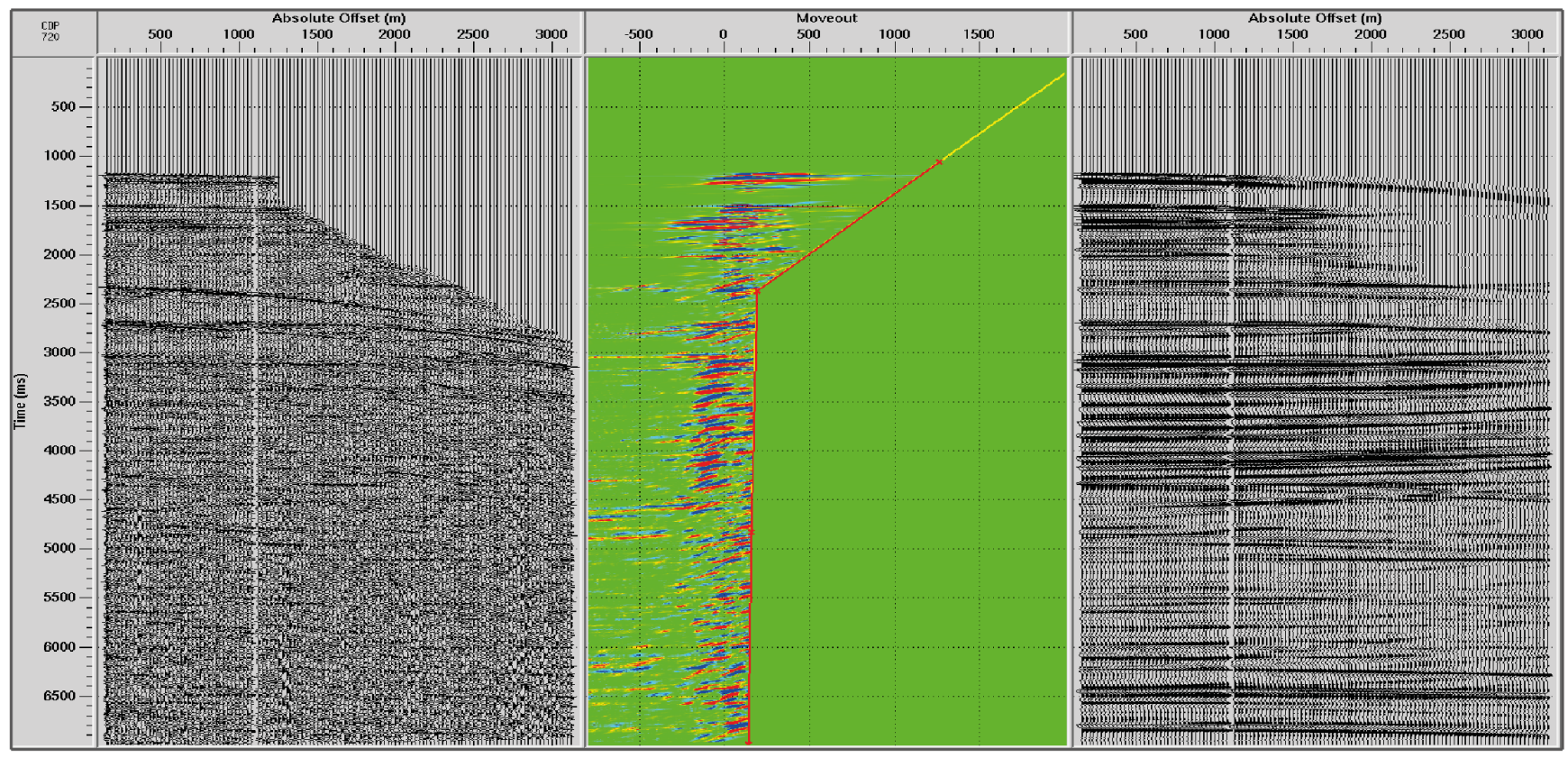

Figure 6: CMP 720 com corrigido cde NMO e seu respectivo espectro Radon com polígono de atenuação exposto. 


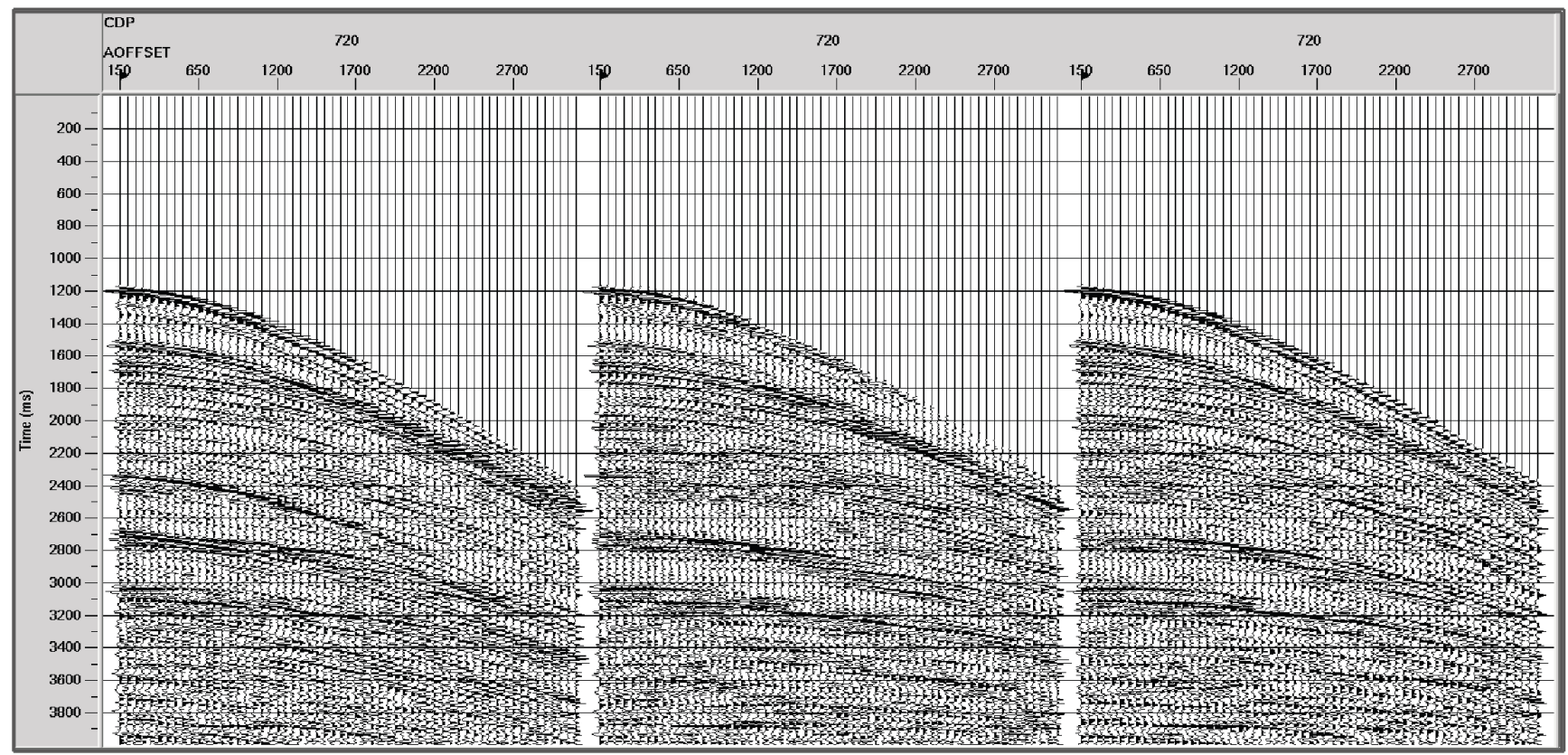

(A)

(B)

(C)

Figure 7: CMP 720: (a) original; (b) filtrado com f-k; (c) filtrado com transformada Radon.

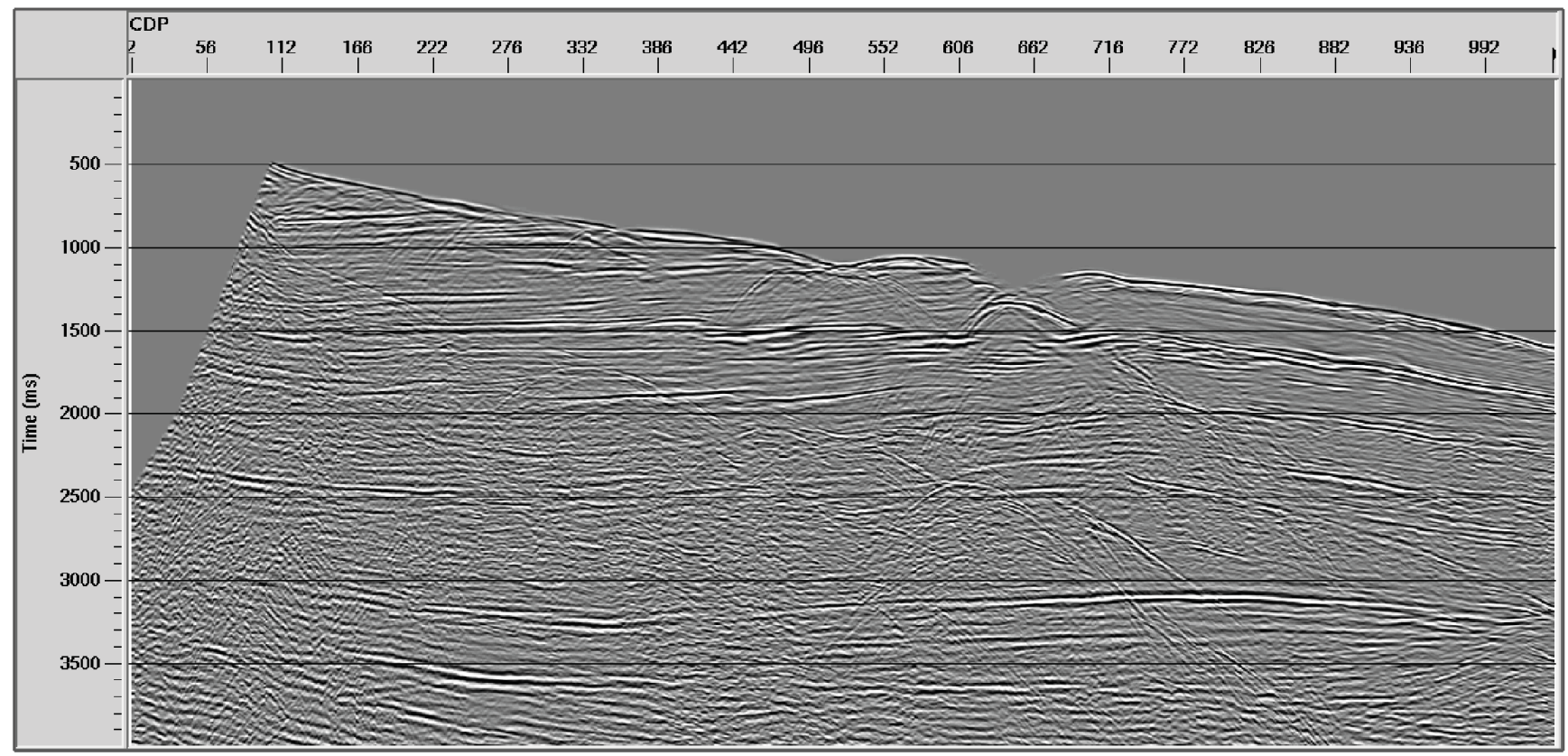

Figure 8: Seção empilhada original. 


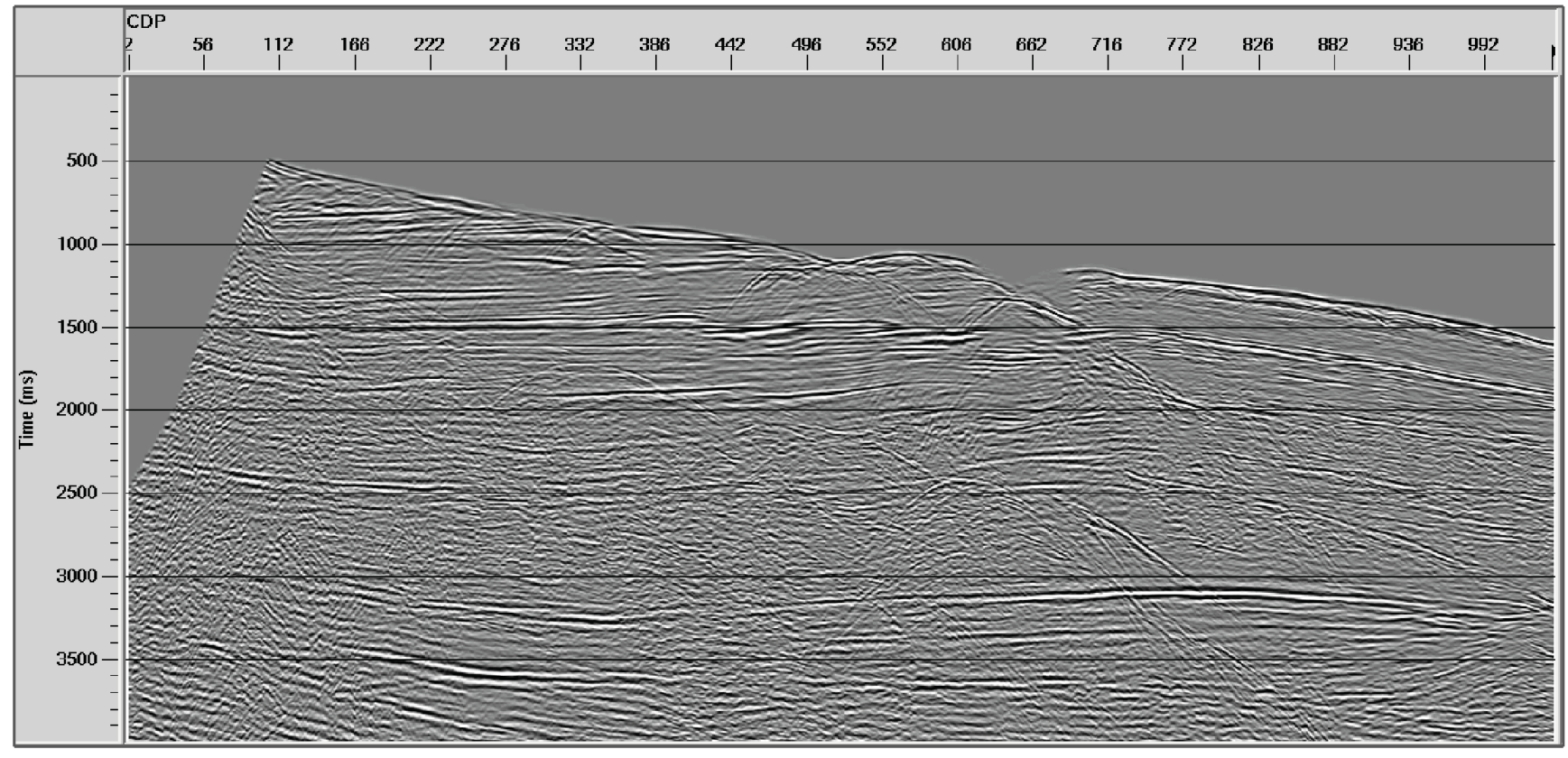

Figure 9: Seção empilhada após a aplicação da filtragem f-k.

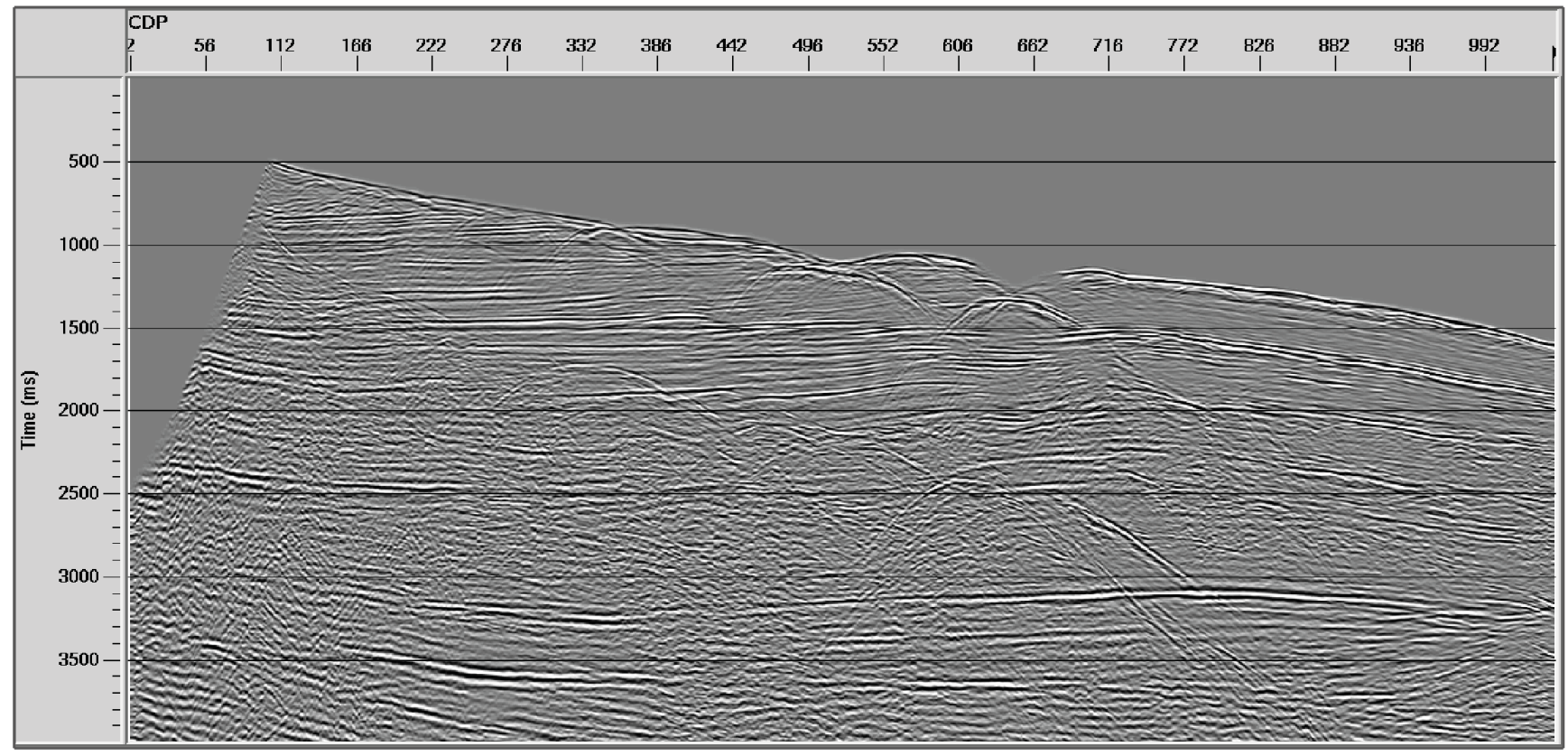

Figure 10: Seção empilhada após a aplicação da filtragem Radon. 